\title{
Transjugular Intrahepatic Portosystemic Shunt Occlusion Complicated with Biliary Fistula Successfully Treated with a Stent Graft: A Case Report
}

\author{
Eunyoung Kim, ${ }^{1}$ Sung Won Lee, ${ }^{1, *}$ Woo Hyeon Kim, ${ }^{1}$ Si Hyun Bae, Nam Ik Han, Jung Suk Oh, \\ Ho Jong Chun, ${ }^{2}$ and Hae Giu Lee ${ }^{2}$
}

${ }^{1}$ Department of Internal Medicine, College of Medicine, The Catholic University of Korea, Seoul, Republic of Korea

${ }^{2}$ Department of Radiology, College of Medicine, The Catholic University of Korea, Seoul, Republic of Korea

${ }^{*}$ Corresponding author: Sung Won Lee, Division of Hepatology, Department of Internal Medicine, College of Medicine, The Catholic University of Korea, Seoul, Republic of Korea. Tel: +82-222582075, Fax: +82-255865589, E-mail: swleehepa@gmail.com

Received 2015 April 5; Revised 2015 August 24; Accepted 2015 October 7.

\begin{abstract}
A 43-year-old man with liver cirrhosis received transjugular intrahepatic portosystemic shunt(TIPS) for the treatment of recurrent variceal bleeding and F3 esophageal varices. During routine follow up liver ultrasound examination, six months after the implantation, TIPS occlusion was suspected and TIPS revision was performed. During the revision, moderate to severe stenosis at the hepatic venous segment of the tract and a total occlusion at the parenchymal segment of TIPS tract near the portal vein with biliary-TIPS fistula were identified with a clear visualization of the common bile duct. After the successful TIPS revision with the placement of an additional stent-graft, the biliary fistula and common bile duct were no more delineated. We herein report a rare case with an obvious visualization of biliary-TIPS fistula associated with obstruction of TIPS shunt on the tractogram and recanalization with an additional stent-graft.
\end{abstract}

Keywords: Stenosis, Fistula, Portosystemic, Transjugular Intrahepatic

\section{Introduction}

Transjugular intrahepatic portosystemic shunt (TIPS) is an effective way to decompress portal hypertension. Due to the high rate of stenosis and occlusion of the TIPS stent, patients are recommended to receive routine follow-up imaging surveillances, such as Doppler ultrasonography, every three to six months. TIPS dysfunction is an important issue with no clear recommendation regarding the use of prophylactic medications to prevent the formation of thrombus or stenosis. Accumulating histologic evidences suggest that the transection of bile ducts during the TIPS procedure and the formation of biliary fistula are major etiologies of the shunt occlusion (1, 2). However, only very few cases of TIPS-associated biliary fistula, which were identifiable with imaging modalities, have been reported. We describe a case of TIPS occlusion complicated with biliary fistula, which was revised successfully by the re-implantation of a stent-graft.

\section{Case Presentation}

A 43-year-old male patient had undergone the TIPS procedure during May 2012 at the Seoul St Mary's hospital for the treatment of recurrent variceal bleeding and F3 esophageal varices. Transjugular intrahepatic portosystemic shunt was created with an $8 \mathrm{~mm} \times 7.5 \mathrm{~cm}$ polytetrafluoroethylene(PTFE)-covered stent(Seal bifurcated stent graft extension; S\&G Biotech, Seongnam-si, Korea) per the standard protocol, between the right portal vein and the right hepatic vein. The stent-graft had bare segments; 5 $\mathrm{mm}$ at the hepatic vein side and $2 \mathrm{~cm}$ at the portal vein side. During the eight months after the TIPS placement, variceal bleeding did not occur. However, in January 2013, the patient's follow-up liver ultrasound examination showed a cut-off in the blood flow within the TIPS stent, and the liver computed tomography (CT) exam revealed a thrombotic occlusion within the TIPS stent with the thrombus extending to the right portal vein. Esophagogastroduodenoscopy also showed the progression of esophageal varices. Therefore, the patient was admitted for TIPS revision. On arrival, the patient was asymptomatic and his mental status was alert. His body temperature was $36.5^{\circ} \mathrm{C}$, blood pressure $120 / 70 \mathrm{~mm} \mathrm{Hg}$, pulse rate $68 /$ minute and respiratory rate 20/minute. The Child-Pugh score was five (class A). Serum aspartate aminotransferase (AST) was $30 \mathrm{U} / \mathrm{L}$, alanine aminotransferase (ALT) 20 $\mathrm{U} / \mathrm{L}$, total bilirubin $0.59 \mathrm{mg} / \mathrm{dL}$, direct bilirubin $0.24 \mathrm{mg} /$ $\mathrm{dL}$, alkaline phosphatase $179 \mathrm{IU} / \mathrm{L}$, total protein $6.8 \mathrm{~g} / \mathrm{dL}$, albumin $3.7 \mathrm{~g} / \mathrm{dL}$, prothrombin time 14.4 seconds (INR 1.34), white blood cell count (WBC) 1160/uL, hemoglobin $11.5 \mathrm{~g} / \mathrm{dL}$, and platelets $46000 / \mathrm{uL}$.

Venogram of the shunt revealed communication be-

Copyright ( ) 2016, Tehran University of Medical Sciences and Iranian Society of Radiology. This is an open-access article distributed under the terms of the Creative Commons Attribution-NonCommercial 4.0 International License (http://creativecommons.org/licenses/by-nc/4.0/) which permits copy and redistribute the material just in noncommercial usages, provided the original work is properly cited. 
Kim E et al.

tween the TIPS tract and the bile duct demonstrating a biliary-TIPS fistula combined with a focal stenosis of the stent at the hepatic venous segment of the TIPS tract (Figure 1). A very slow flow was detected within the TIPS tract and the right portal vein was not opacified. After negotiation of the occluded segment and the right portal vein with a 0.035 hydrophilic guide wire (Terumo, Japan) and $4 \mathrm{~F}$ cobra catheter (Terumo, Japan), the catheter was placed in the main portal vein through the struts of the portal side bare segment. On the portal venogram, there was a partial filling defect, which extended from the proximal right portal vein to the posterior segmental portal vein that contained the stent graft. The increased portosystemic pressure gradient $(27 \mathrm{mmHg}$ ) was measured. The occluded segment was dilated with an $8 \mathrm{~mm}$ balloon catheter, and an $8 \mathrm{~mm} \times$ $10 \mathrm{~cm}$ PTFE-covered stent (S\&G Biotech) was implanted in a stent-in-stent manner. The stent-graft was placed between the proximal right portal vein and the right hepatic vein near the opening of the inferior vena cava. Following the stent-graft placement, the venogram showed restoration of the blood flow in the TIPS tract, and the biliary fistula was no longer visualized. Slow flow in the peripheral portal veins and the coronary veins were observed without residual stenosis (Figure 2). Final portosystemic pressure gradient decreased to $15 \mathrm{mmHg}$. Moderate stenosis of the proximal right portal vein persisted as a filling defect on the completion portogram. Two days later, the patient was discharged from the hospital without any immediate complication. Doppler sonography six weeks after the TIPS revision showed patency of the TIPS tract. Flow velocity of the portal vein and the hepatic vein was measured to be 40 $\mathrm{cm} /$ second and $120 \mathrm{~cm} /$ second, respectively.

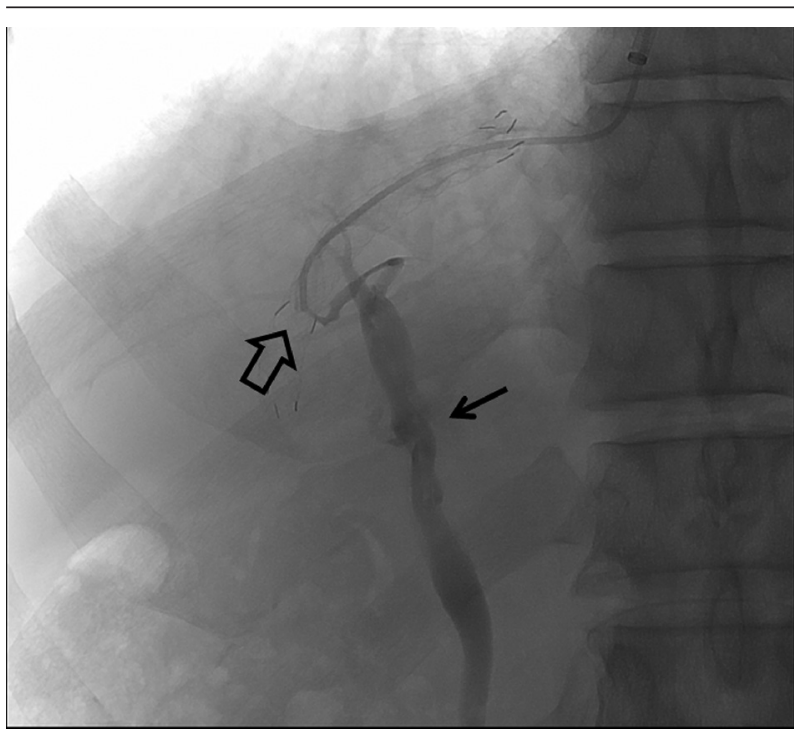

Figure 1. A 43-year-old cirrhotic man with TIPS occlusion complicated with biliary fistula. Venogram through the right internal jugular access. Total occlusion was observed at the parenchymal segment of the TIPS tract (open arrow). Transjugular Intrahepatic Portosystemic Shunt-biliary fistula with contrast leakage to the common bile duct was delineated (black arrow).

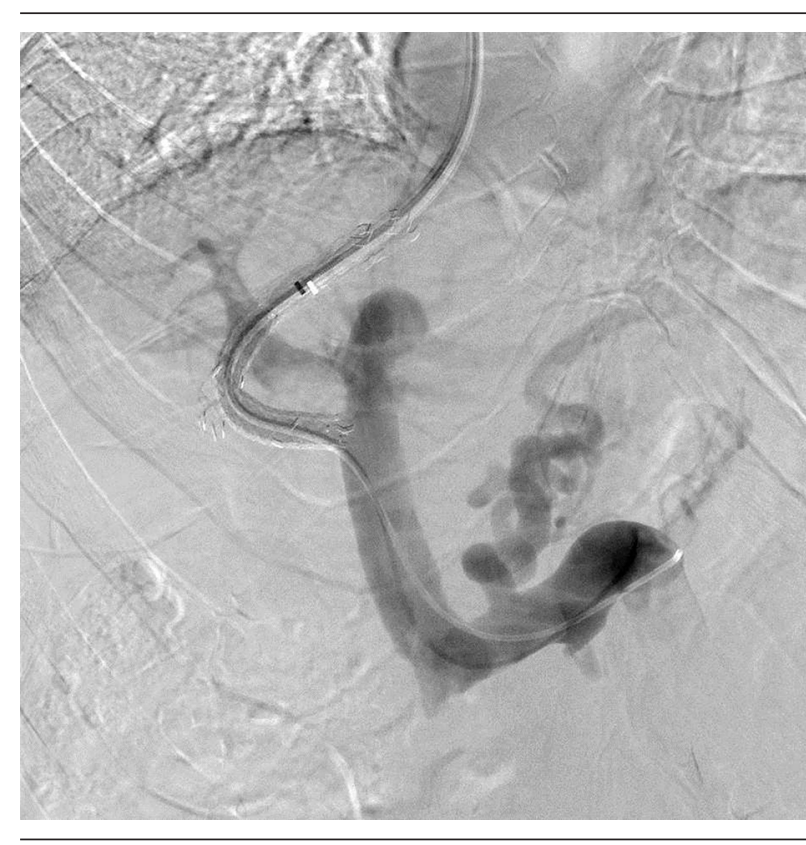

Figure 2. Post-procedure portal venogram; the image shows the restoration of the shunt function after balloon angioplasty at the hepatic venous segment and an additional stent-graft. Transjugular intrahepatic portosystemic shunt-biliary fistula is no more visualized.

\section{Discussion}

TIPS is an effective treatment for reducing portal hypertension. It is applied for cases of recurrent variceal bleeding, refractory ascites, Budd-Chiari syndrome and clinically as a bridge to liver transplantation. Garicia-Pagan et al. reported that the use of early-TIPS for patients at highrisk of variceal bleeding resulted in a much lower incidence of bleeding control failure (3) and also reported that early TIPS was more effective than endoscopic treatment in preventing variceal re-bleeding and improving survival rate (3). However, long-term efficacy of TIPS is limited due to shunt stenosis or occlusion. LaBerge et al. reported that the rates of stenosis and occlusion within two years after TIPS were approximately $47 \%$ and $12 \%$, respectively (4).

The TIPS procedure creates communication between the portal system and the systemic circulation via a percutaneous approach with penetration of the liver parenchyma. In the process, injury may develop in the structures adjacent to the targeted portal vein including the hepatic parenchyma or the portal triad, which consists of the portal vein, hepatic artery and bile duct. In cases of significant bile duct injury, TIPS-biliary fistula may develop resulting in bile leakage and ultimately TIPS occlusion (1).

The frequent locations of the TIPS stenosis are the hepatic venous end of the stent and the parenchymal portion (5). It has been reported that there is a histological difference between the two locations in case of stenosis due to different etiologies. Narrowings at the outflow 
tract of the hepatic vein were usually associated with intimal hyperplasia as a consequence of shear stress and turbulence from increased high-velocity blood flow (1, $6)$. In cases of mid-shunt stenosis, "pseudo-intimal hyperplasia" with the formation of granulation tissue and thickening of the neointima, composed of myofibroblasts and collagen, were usually observed. Szeet et al. reported that "pseudo-intimal hyperplasia" was closely related to bile duct injury (1). LaBerge et al. reported that biliary staining was associated with exuberant inflammation and granulation tissue replacement (6). Meanwhile, LaBerge et al. (6) suggested another theory on the association between bile leakage and the development of TIPS stenosis. Bile is considered thrombogenic because it is rich in bile acids, bile salts, cholesterol, and phospholipid and thus, by stimulating coagulation cascade, biliary fistula may lead to acute thrombosis. Accordingly, a case of acute TIPS occlusion nine hours after the TIPS procedure due to the identifiable biliary-to-TIPS fistula formation was reported (2).

In our case, stenosis developed at the hepatic venous segment and total occlusion with biliary fistula formation developed at the portal side of the stent-graft. The stenosis and the occlusion were each located at both ends of the covered segments of the stent. From this, it may be hypothesized that the insufficiently covered hepatic venous segment by the PTFE graft was related to stenosis and the exposure of the TIPS tract to bile acids due to the biliary fistula formation led to stent occlusion. Also, shortening of the stent-graft, which has a similar mesh structure with wallstents (Boston scientific), may have been related to the events; this explains the late development, eight months after the shunt creation, of the stenosis and the occlusion (7).

Shunt recanalization by angioplasty and re-stenting is regarded as a standard option in the revision of shunt occlusion. In case of biliary-to-TIPS fistula, there is no established recommendation regarding the type of stent yet generally covered stents are favored over bare stents. Tanaka et al. reported that with PTFE-covered stent grafts, the rate of in-stent stenosis or stent occlusion within the first year after TIPS dropped significantly compared to the bare metal stents (8). Jung et al. reported that TIPS created with expanded PTFE stent-grafts showed not only superior primary patency rates but also clinical outcomes defined as better control of bleeding and ascites compared with bare stents (9). These results may be related to the fact that covered stents can almost completely prevent bile from entering the stent lumen (10).

In summary, accumulating evidence supports the theory that biliary spillage is one of the major etiologies of the mid-shunt occlusion. In our case, stent occlusion complicated with clearly visualized biliary fistula was detected eight months after the placement with combined stenosis at the hepatic venous segment. The patient was successfully treated with angioplasty at the hepatic venous segment and implantation of a covered stent in a stent-in-stent manner. Revision using bare stents or angioplasty alone may be effective in some cases yet for the treatment of stent occlusion with proven biliary fistula, revision using covered stents seems to be advantageous in preventing permeation of the bile into the stent.

\section{Acknowledgments}

None declared.

\section{Footnotes}

Authors' Contribution:Eunyoung Kim, Sung Won Lee and Woo Hyeon Kim wrote the manuscript. The manuscript was edited under the supervision of Si Hyun Bae and Nam Ik Han. Jung Suk Oh, Ho Jong Chun and Hae Giu Lee performed the TIPS revision and gave advice on the radiologic aspects of this case.

Financial Disclosure:The authors have nothing to disclose

Funding/Support:None declared.

\section{References}

1. Sze DY, Vestring T, Liddell RP, Kato N, Semba CP, Razavi MK, et al. Recurrent TIPS failure associated with biliary fistulae: treatment with PTFE-covered stents. Cardiovasc Intervent Radiol. 1999;22(4):298-304. [PubMed: 10415219]

2. Boyvat F, Cekirge S, Balkanci F, Besim A. Treatment of a TIPS-biliary fistula by stent-graft in a 9-year-old boy. Cardiovasc Intervent Radiol.1999;22(1):67-8. [PubMed: 9929548]

3. Garcia-Pagan JC, Di Pascoli M, Caca K, Laleman W, Bureau C, Appenrodt $B$, et al. Use of early-TIPS for high-risk variceal bleeding: results of a post-RCT surveillance study. J Hepatol. 2013;58(1):4550. doi: 10.1016/j.jhep.2012.08.020. [PubMed: 22940408]

4. LaBerge JM, Somberg KA, Lake JR, Gordon RL, Kerlan RK, Ascher $\mathrm{NL}$, et al. Two-year outcome following transjugular intrahepatic portosystemic shunt for variceal bleeding: results in 90 patients. Gastroenterology. 1995;108(4):1143-51. [PubMed: 7698582]

5. Saxon RS, Ross PL, Mendel-Hartvig J, Barton RE, Benner K, Flora $\mathrm{K}$, et al. Transjugular intrahepatic portosystemic shunt patency and the importance of stenosis location in the development of recurrent symptoms. Radiology. 1998;207(3):683-93. doi: 10.1148| radiology.207.3.9609891. [PubMed: 9609891]

6. LaBerge JM, Ferrell LD, Ring EJ, Gordon RL. Histopathologic study of stenotic and occluded transjugular intrahepatic portosystemic shunts. J Vasc Interv Radiol. 1993;4(6):779-86. [PubMed: 8281000]

7. Tesdal IK, Jaschke W, Buhler M, Adamus R, Filser T, Holm E, et al. Transjugular intrahepatic portosystemic shunting (TIPS) with balloon-expandable and self-expanding stents: technical and clinical aspects after $31 / 2$ years' experience. Cardiovasc Intervent Radiol.1997;20(1):29-37. [PubMed: 8994721]

8. Tanaka T, Gunther RW, Isfort P, Kichikawa K, Mahnken AH. Pullthrough technique for recanalization of occluded portosystemic shunts (TIPS): technical note and review of the literature. Cardiovasc Intervent Radiol. 2011;34(2):406-12. doi: 10.1007/s00270-0109874-2. [PubMed: 20440498]

9. Jung HS, Kalva SP, Greenfield AJ, Waltman AC, Walker TG, Athanasoulis CA, et al. TIPS: comparison of shunt patency and clinical outcomes between bare stents and expanded polytetrafluoroethylene stent-grafts. J Vasc Interv Radiol. 2009;20(2):180-5. doi: 10.1016/j.jvir.2008.11.005. [PubMed:19097918]

10. HaskalZJ, Davis A, McAllister A, Furth EE. PTFE-encapsulated endovascular stent-graft for transjugular intrahepatic portosystemic shunts: experimental evaluation. Radiology. 1997;205(3):682-8. doi:10.1148/radiology.205.3.9393521. [PubMed: 9393521] 